\title{
Global characteristics of the first IBIS/ISGRI catalogue sources: unveiling a murky episode of binary star evolution
}

\author{
A. J. Dean ${ }^{1}$, A. Bazzano ${ }^{2}$, A. B. Hill ${ }^{1}$, J. B. Stephen ${ }^{3}$, L. Bassani ${ }^{3}$, E. J. Barlow ${ }^{1}$, A. J. Bird ${ }^{1}$, F. Lebrun ${ }^{4}$, V. Sguera ${ }^{1}$, \\ S. E. Shaw ${ }^{1,5}$, P. Ubertini ${ }^{2}$, R. Walter ${ }^{5}$, and D. R. Willis ${ }^{1, \star}$
}

1 School of Physics and Astronomy, University of Southampton, Highfield, SO17 1BJ, UK

e-mail: ajd@astro.soton.ac.uk

2 IASF - Rm, INAF, via Fosso del Cavaliere 100, 00133 Rome, Italy

3 IASF - Bo, INAF, via Gobetti 101, 40129 Bologna, Italy

4 CEA - Saclay, DAPNIA/Service d'Astrophysique, 91191 Gif-sur-Yvette Cedex, France

5 Geneva Observatory, INTEGRAL Science Data Centre, Chemin d'Ecogia 16, 1291 Versoix, Switzerland

Received 25 May 2005 / Accepted 2 August 2005

\section{ABSTRACT}

INTEGRAL is the first gamma-ray astronomy mission with a sufficient sensitivity and angular resolution combination appropriate to the detection and identification of considerable numbers of gamma-ray emitting sources. The large field of view ( 30 zero response FWHM) enables INTEGRAL to survey the galactic plane on a regular ( $\sim$ weekly) basis as part of the core programme. The first source catalogue, based on the 1st year of core programme data ( $\sim 5 \mathrm{Ms})$ has been completed and published (Bird et al. 2004, ApJ, 607, L33). It contained $123 \gamma$-ray sources (24 HMXB, 54 LMXB, 28 "unknown", plus 17 others) - sufficient numbers for a reasonable statistical analysis of their global properties. These were located to a positional accuracy of typically $0.72 \mathrm{arcmin}$. The detection of previously unknown $\gamma$-ray emitting sources generally exhibiting high intrinsic absorption, which do not have readily identifiable counterparts at other wavelengths, is intriguing. The substantial fraction (roughly $20 \%$ of the total number) of unclassified $\gamma$-ray sources suggests they must constitute a significant family of objects. In this paper we review the global characteristics of the known galactic sources as well as the unclassified objects with the twin aims of investigating how the unclassified set may fit into stellar evolution and improving our understanding of known X-ray binary systems through the non-thermal $\gamma$-ray channel. In the context of the known systems we are very conscious that they constitute a $\gamma$-ray selected set, and may exhibit subtle generic differences to the rest of the class. We present $\log (N)-\log (S)$ distributions, angular distributions, and for systems with reliable distance estimates the spatial distributions within the Galaxy and luminosity functions. For the unknown sources, this statistical analysis has shown that they are most likely to be HMXBs containing a highly magnetised neutron star. The lack of X-ray counterparts for these sources indicates a high degree of intrinsic obscuration.

Key words. gamma-rays: observations - X-rays: binaries - Galaxy: general - Galaxy: structure - Galaxy: stellar content

\section{Introduction and the first IBIS/ISGRI catalogue}

INTEGRAL is the first gamma-ray astronomy mission with a sufficient sensitivity and angular resolution combination appropriate to the detection and identification of considerable numbers of gamma-ray emitting sources (Winkler et al. 2003). With an observation time of roughly $1000 \mathrm{~s}$ required to detect a $1 M_{\odot}$ neutron star emitting at the Eddington limit from a distance of $10 \mathrm{kpc}$ it is clear that a meaningful survey of discrete galactic gamma-ray emitting objects is possible in the lifetime of INTEGRAL. These will be located to a positional accuracy of typically 0.72 arcmin.

A significant fraction of the INTEGRAL core programme is devoted to regular scans of the galactic plane and a deep exposure of the galactic centre. This is facilitated by the large

^ Present address: INTEGRAL Science Data Centre, Chemin d'Ecogia 16, 1290 Versoix, Switzerland. field of view of the on-board telescopes $\left(9^{\circ} \times 9^{\circ}\right.$ fully coded and $29^{\circ} \times 29^{\circ}$ zero response for IBIS, Ubertini et al. 2003), which permits $\sim 10^{4} \mathrm{~s}$ exposures of the galactic plane to be made on a regular basis ( every 12 days). The sample of sources used for this study is derived directly from the first IBIS/ISGRI catalogue (Bird et al. 2004), which represents the first year of the INTEGRAL Galactic Plane Survey (GPS) and Galactic Centre Deep Exposure (GCDE) observations. The 1st IBIS/ISGRI survey catalogue was compiled based on all Core Programme observations between revolutions 46 and 120 inclusive. INTEGRAL/IBIS data is organised into short pointings (science windows) of $2000 \mathrm{~s}$. OSA software version 3 was used to create images at science window level, including two iterations of processing to first produce a cleaning catalogue, and a second processing to remove all ghosts from the images. The $\sim 2500$ individual science window images were then mosaiced using a custom tool to produce deep all-sky maps. An 
initial source list was obtained by searching all-sky mosaics constructed in both $20-40$ and $30-50 \mathrm{keV}$ bands, using the SExtractor tool. The $20-40 \mathrm{keV}$ band was optimised for detection of soft sources, and a threshold of 7 sigma was applied. The 30-50 keV band was judged to produce the cleanest images, and a threshold of 6 sigma was used. By inspection of the image statistics, it was determined that at maximum, one source in the catalogue would be a false detection. It has since been discovered that the indentification of IGR J17460-3047 was in fact a false detection.

Positions for each source were provided using the centroiding function in SExtractor, which allows for a non-Gaussian PSF imposed by searching all-sky maps in the Aitoff projection. The source positions were principally extracted from the 30-50 keV maps, which were least contaminated by background structures. In the case of very soft sources, positions were taken from the $20-40 \mathrm{keV}$ maps. Positions for previously known sources were cross-checked against archive positions, and found to match the expected point source location accuracy well (Gros et al. 2003). The typical point source location error range is $20^{\prime \prime}-3^{\prime}$, with a 1 arcmin error circle for a source $>10 \sigma$ (90\% confidence). The precision of the source locations allow for the clear identification/association to sources seen by previous missions and hence source classification is possible. Additionally, those sources without previously seen high energy counterparts can be confidently identified as newly discovered INTEGRAL $\gamma$-ray sources. Fluxes for each source were obtained by extraction of values from the all-sky mosaics at the positions of best fit provided by SExtractor.

The 1st IBIS/ISGRI survey catalogue is constructed from approximately $5 \mathrm{Ms}$ of observations spread throughout the period February 2003 to October 2003. The observations were clustered along the galactic plane; consequently most of the $\gamma$-ray sources are stellar systems in one form or another. INTEGRAL observes the available galactic longitude in a sunorthogonal manner dictating that most regions of the galactic plane have been observed intermittently during two typically three month periods within the annual cycle and spaced roughly six months apart. Since most of the galactic $\gamma$-ray sources are highly variable the catalogue is a time averaged map and the flux value quoted depends on its total accumulated flux averaged over the period of the observation for that region of the sky, no other attempt has been made to correct the recorded flux to an "average" value. A number of transient objects are therefore missed if their cumulative emission over the survey period does not rise above the aggregate threshold.

Of the 123 sources in the catalogue, 5 were unambiguously identified with AGN, 5 with white dwarfs, 4 with radio pulsars (free neutron stars), 3 supernovae remnants and one cluster. Of the remainder, 54 were identified with known low mass $\mathrm{X}$-ray binary (LMXB) systems, 24 with high mass X-ray binarys (HMXB) and the remaining 28 sources have no firm classification and are here after described as "unclassified". In this paper we have only considered the LMXBs, the HMXBs and the unclassified objects, discounting IGR J17460-3047 as a false detection; the small numbers of the remaining object types preclude any meaningful statistical analysis. Formally, the LMXBs were classified as having the primary star with a mass $<1 M_{\odot}$ (Liu et al. 2001) and HMXBs having a primary star with $M>1 M_{\odot}$ (Liu et al. 2001). Since the distribution of the unclassified sources is strongly associated with the galactic structure we have assumed, for the purposes of deriving $\log N-\log S$ distributions that the contamination of the sample by extragalactic sources is extremely small, or even non existent.

The number of galactic gamma-ray sources in the first IBIS-ISGRI catalogue are comparable to the early X-ray catalogues e.g. Warwick et al. (1981) and are sufficient for a preliminary investigation of their global characteristics on a statistical basis. It should be noted that the sources presented in the IBIS-ISGRI catalogue are gamma-ray selected and, although many of them are well known X-ray objects, it is not surprising that there are considerable differences to catalogues of X-ray sources of corresponding depth. This is due to the fact that IBIS-ISGRI detects photons at energies largely unaffected by photoelectric absorption in universal abundance matter. Hence objects highly photo absorbed in the classical X-ray band are clearly visible at gamma-ray energies, a factor confirmed by the significant fraction of sources previously unclassified. There is clearly a thrust to find out what these unclassified objects actually are and how they fit into the overall picture of stellar evolution. There are two main methods to achieve this end. One is to perform follow-up observations on the individual gamma-ray sources at other wavelengths in order to identify a counterpart and thus permit more detailed studies, where possible, over a wider spectral range. The other approach is to study the sample on a statistical basis and compare their overall characteristics with other known generic sets of astronomical objects. There is a strong degree of complementarity in the two methods; here we investigate the global characteristics of the $\gamma$-ray sample.

\section{The $\log (N)-\log (S)$ distributions}

In this section we construct the number flux distribution for the various source types. We have assumed the usual power-law form of $N(>S)=K S^{-\alpha}$ for the relationship. Figure 1 shows the raw form of these relationships for all galactic sources, the HMXB, the LMXB and the unclassified sources separately. The source intensities have been converted into $20-40 \mathrm{keV}$ Crab equivalent units. The decrease in source density below a few milliCrab is due to the flattening of the absolute source distribution as the sample is flux limited around that value. This corresponds to an energy flux of $\sim 3 \times 10^{-11} \mathrm{erg} \mathrm{s}^{-1} \mathrm{~cm}^{-2}$ for a Crab-like spectrum.

As the sky coverage of the first survey is not complete, these curves are only indicative of the true galactic distributions and must be corrected for both the area of sky covered and the depth of exposure (or the minimum detectable flux (MDF)) at each point. This is not straightforward due to the residual systematic structures in the sky maps, however it is possible by inspection of the error and exposure distributions to parameterise the variation in MDF as a function of exposure, thereby taking into account the effects of the residual systematic variations.

From this relationship and the general sky exposure map we can then construct Fig. 2 which shows the area of sky (in 


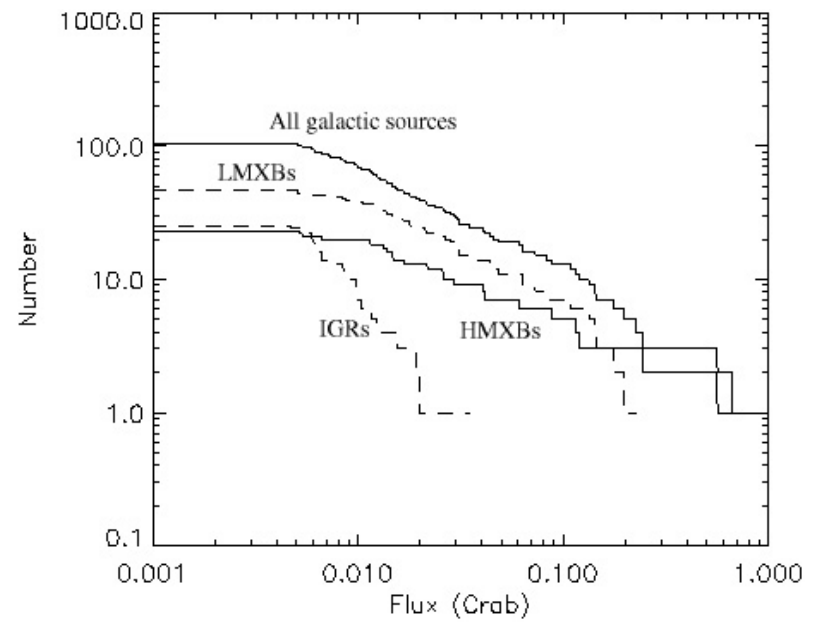

Fig. 1. The raw Number-Flux relationship for the sources in the 1st INTEGRAL/IBIS survey. The curves shown are for, All Galactic Sources; LMXB's, HMXB's and the unclassified sources.

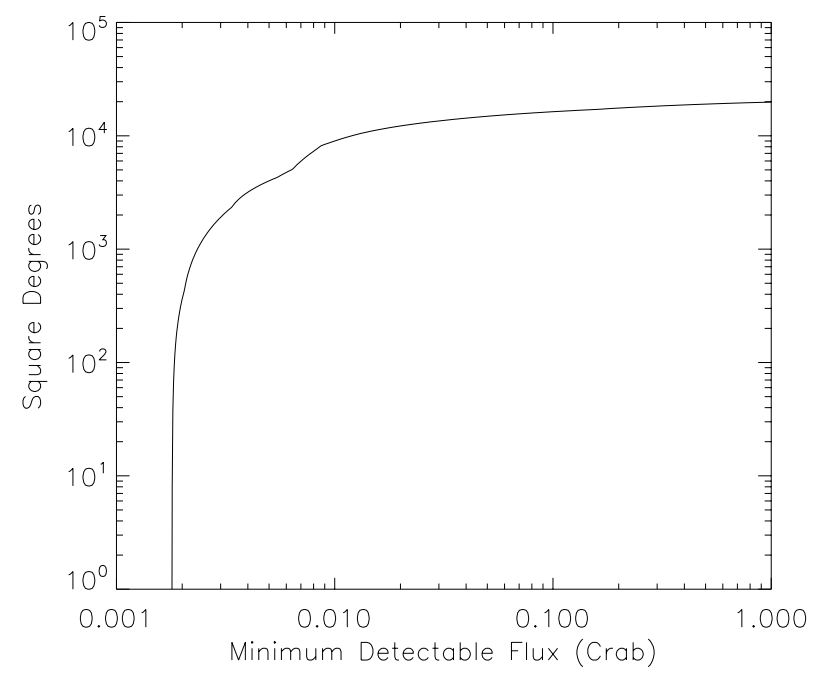

Fig. 2. The area of sky observed in the survey as a function of the minimum detectable flux (6 sigma). The flux is in equivalent Crab units.

square degrees) observed as a function of MDF (6 sigma in Crab units).

We can then use this curve to correct the observed $\log N-\log S$ resulting in the relationships shown in Fig. 3.

The limiting detection threshold of 6 sigma is sufficiently high so that the maximum likelihood (ML) method (Murdoch \& Crawford 1973) can be used to calculate the best-fit values of the slope of the number-flux relationship without the uncertainty in the correction factor for weaker sources dominating the correction itself. This has been performed for both the uncorrected and corrected distributions and the value of the slope found in each case is shown in Table 1.

It is of interest to consider and compare the $\log (N)-\log (S)$ distributions of the various sub-groupings. The -0.91 slope of the power law for all the combined galactic sources is close to the -1 value expected for a uniform infinite plane distribution and lies between the value $(-0.79 \pm 0.07)$ measured for

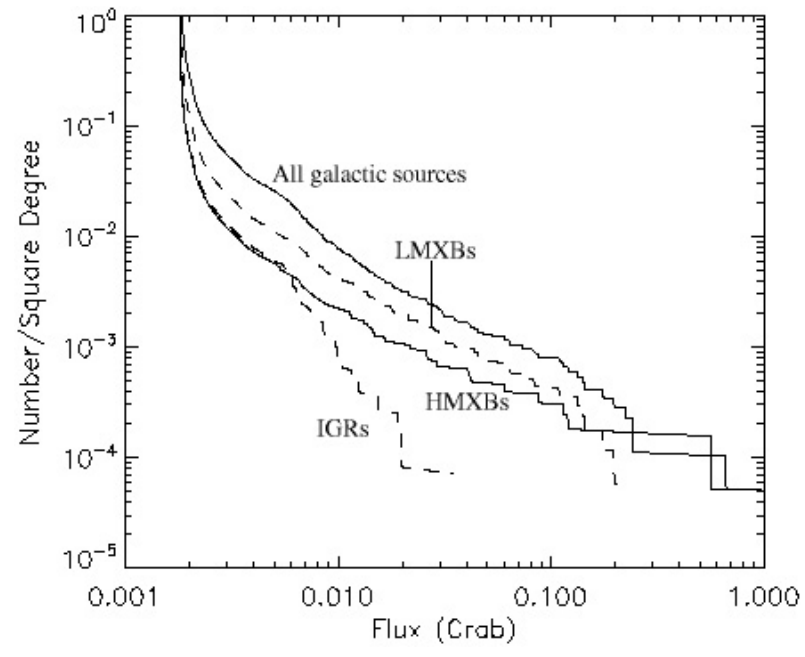

Fig. 3. The Number-Flux relationships as shown in Fig. 1 but corrected for exposure and sky area observed.

Table 1. Slope of Number/Flux relation.

\begin{tabular}{ccc}
\hline \hline Source class & Uncorrected slope & Corrected slope \\
\hline All Galactic Sources & $-0.81 \pm 0.10$ & $-0.91 \pm 0.09$ \\
LMXB & $-0.81 \pm 0.13$ & $-0.95 \pm 0.13$ \\
HMXB & $-0.65 \pm 0.15$ & $-0.81 \pm 0.15$ \\
Unclassified Sources & $-1.79 \pm 0.37$ & $-2.11 \pm 0.46$ \\
\hline
\end{tabular}

galactic sources by ASCA (Sugizaki et al. 2001) in the classic $2-10 \mathrm{keV} \mathrm{X}$-ray band and the $(-1.1)$ slope derived from the Einstein galactic plane survey (Hertz \& Grindlay 1984). It is significantly steeper than the value $(\sim-0.5)$ derived for the bright galactic sources from the Ariel 5 catalogue (Warwick et al. 1981). This all-source value is clearly dominated by the numerically superior LMXBs, which when detached provide a slope of $-0.95 \pm 0.13$ as a sub-set. The value for LMXBs is thus consistent with -1 , as qualitatively expected for a population with a larger scale height (Sugizaki et al. 2001) and less aligned with the spiral arm tubes. In contrast HMXBs are typically located within spiral arms and this would automatically lead to a value of $\alpha$ closer to 0.5 . In this context, the $-0.81 \pm 0.13$ slope measured for the HMXBs appears slightly flatter than the LMXBs and may reflect the likely location of these objects along the spiral arms, however the precision of the LMXB and HMXB slopes is insufficient to distinguish between the two groups. The steep slope $(-2.11)$ estimated for the unclassified sources is intriguing and could imply a different source population at low fluxes, this is far steeper than we may expect from even an extragalactic contamination. However, it must be remembered that there are relatively very few of these objects, and they are also amongst the weaker sources in the survey therefore care must be taken in any interpretation of this slope, as indicated by the large uncertainty obtained. Given the angular distribution of the unclassified sample (see Fig. 6), which indicates a concentration towards the central regions of the galaxy it could be argued that this steeper distribution may be due to an increase in their space density towards the galactic centre. 


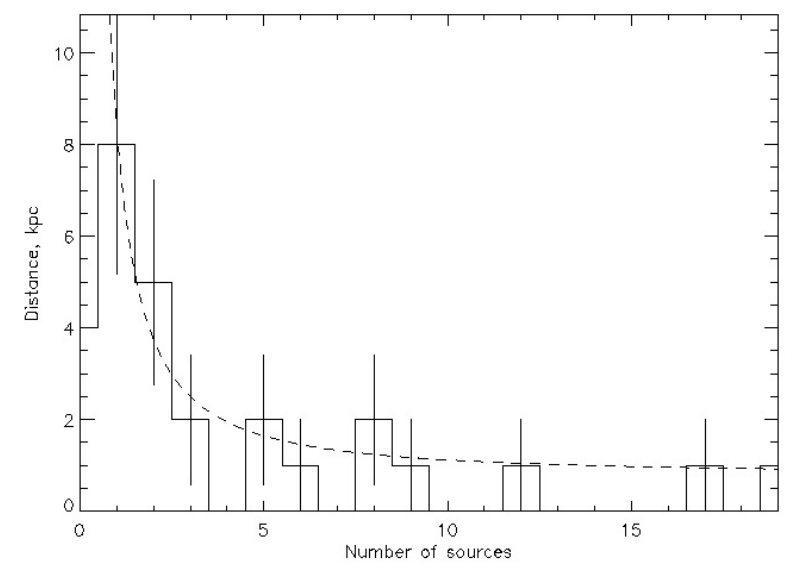

Fig. 4. A histogram of the absolute distance of unclassified sources away from the galactic centre perpendicular to the line of sight (assuming they are $\sim 8 \mathrm{kpc}$ away). The dashed line represents a power-law fit to the histogram.

If we follow this line of reasoning, and if we assume that the newly discovered INTEGRAL unclassified sources are all located in the galactic bulge region we can investigate their spatial distribution by assuming the distribution is the same in the line of sight towards the galactic centre as it is orthogonal to our line of sight. We set the zero point to lie at the peak of the distribution and take absolute values of the distance of sources from this point because of the low number of statistics. The resulting distribution is shown in Fig. 4. The decay in the number of sources away from the Galactic Centre can be fitted by a power law with an index of $\sim 1.3 \pm 0.8$. This distribution, coupled with the fact that the sensitivity of the first catalogue does not extend appreciably beyond the galactic centre region, would imply that for a disk population the sources would obey a $\log N$-Log $S$ distribution with a slope of $1.7 \pm 0.4$, only about 1 standard deviation away from the observed value quoted above. In general due to the limited number of sources in the first catalogue, and also the remaining systematics affecting the imaging process these results are necessarily subject to several sources of error which will be overcome with the release of the second catalogue. Nevertheless they provide qualitative information on the behavious of galactic source populations above $20 \mathrm{keV}$.

\section{The angular distributions}

The distribution of the $\gamma$-ray emitting sources on the sky is shown in Fig. 5. The open circles correspond to the positions of LMXBs, the filled circles to HMXBs, the unclassified sources are represented by the triangles. Superimposed on this sky map are contours of the sky exposure. It is readily apparent that the first year of INTEGRAL observations have been strongly biased towards the study of the Galactic Plane and in particular the Galactic Centre, hence the first IBIS/ISGRI catalogue is essentially a register of galactic sources. Figure 5 also documents the lack of uniformity of the exposure along the Galactic Plane. Despite this non-uniformity, the concentration of the $\gamma$-ray selected HMXBs along the Galactic Plane is clearly apparent, and likewise the grouping of LMXBs in the galactic Bulge region.
Figure 6 reveals the considerable differences in the longitudinal distribution of the HMXB and LMXB sources. Whereas the LMXB are concentrated in the Galactic Centre region, the HMXB are spread more extensively along the Galactic Plane, but in a non-uniform manner, with some evidence for bunching in the vicinity of the tangential points of the spiral arm structure. The locations of the spiral arm tangents were taken from Englmaier et al. (1999). Additionally, a number of HMXBs are seen at $l^{\mathrm{II}} \sim \pm 90^{\circ}$, which is indicative of systems located within our particular spiral arm. A recent study of INTEGRAL observed HMXBs by Lutovinov et al. (2005b) arrives at a similar conclusion. This association had been previously noted through inspection of the Ginga data by Koyama et al. (1990) and in the RXTE/ASM data by Grimm et al. (2002).

The association with the spiral arms is entirely to be expected since the high mass binaries are young stellar systems and should be attached to regions where star formation has recently taken place, such as the spiral arms. We will come back to this point later, when we discuss the spatial distribution of the $\gamma$-ray HMXBs.

Upon first inspection the unclassified INTEGRAL sources appear to have a longitude distribution concentrated around the Galactic Centre, similar to that of the LMXBs. To some extent this is misleading as the Galactic Centre has had the most sky exposure and hence we are more likely to detect new systems in this region. However upon closer examination, whilst some emulate the distribution of LMXB, it can be seen that the unclassified sources do not have a symmetrical distribution about the Galactic Centre and show some tendency to cluster around the locations of spiral arm tangents, specifically the Scutum, 3-kpc and Norma arms, as shown in Fig. 6. Specifically the unclassified sources appear to concentrate in the 3-kpc and Norma spiral arms.

The angular distribution off the Galactic Plane of the sources is seen in Fig. 7. The HMXBs exhibit a much narrower range of angular separations as expected if they are confined to the Galactic Disc. The much wider spread seen in the LMXBs is indicative of a population derived from the Galactic Bulge. Predictably the higher spread in the latitude distribution of the LMXB as opposed to the accumulation of HMXB closer to the Galactic Plane is to be expected from the relative ages of their progenitor stars. The unclassified sources appear to have a sharp latitude cut-off similar to that of the HMXBs, this may indicate that they too are a primarily a population from the Galactic Disc. The unclassified sources, however, do not precisely conform to either of the subgroups; this is not unexpected as they could consist of a mixture of both, or be a separated generic set. Although the longitude distribution is not entirely dissimilar to the LMXBs, this is probably distorted by an effect of exposure and the source concentration around spiral arm tangents suggests an association with high mass systems. This association is further supported by their latitude profile that more closely resembles that of the HMXB. However the unclassified sources are very much associated with the Galactic Centre region, this association may be generic in some way and possibly an artefact of the selection of the sources through the $\gamma$-ray channel, which is not sensitive to the effects of higher 


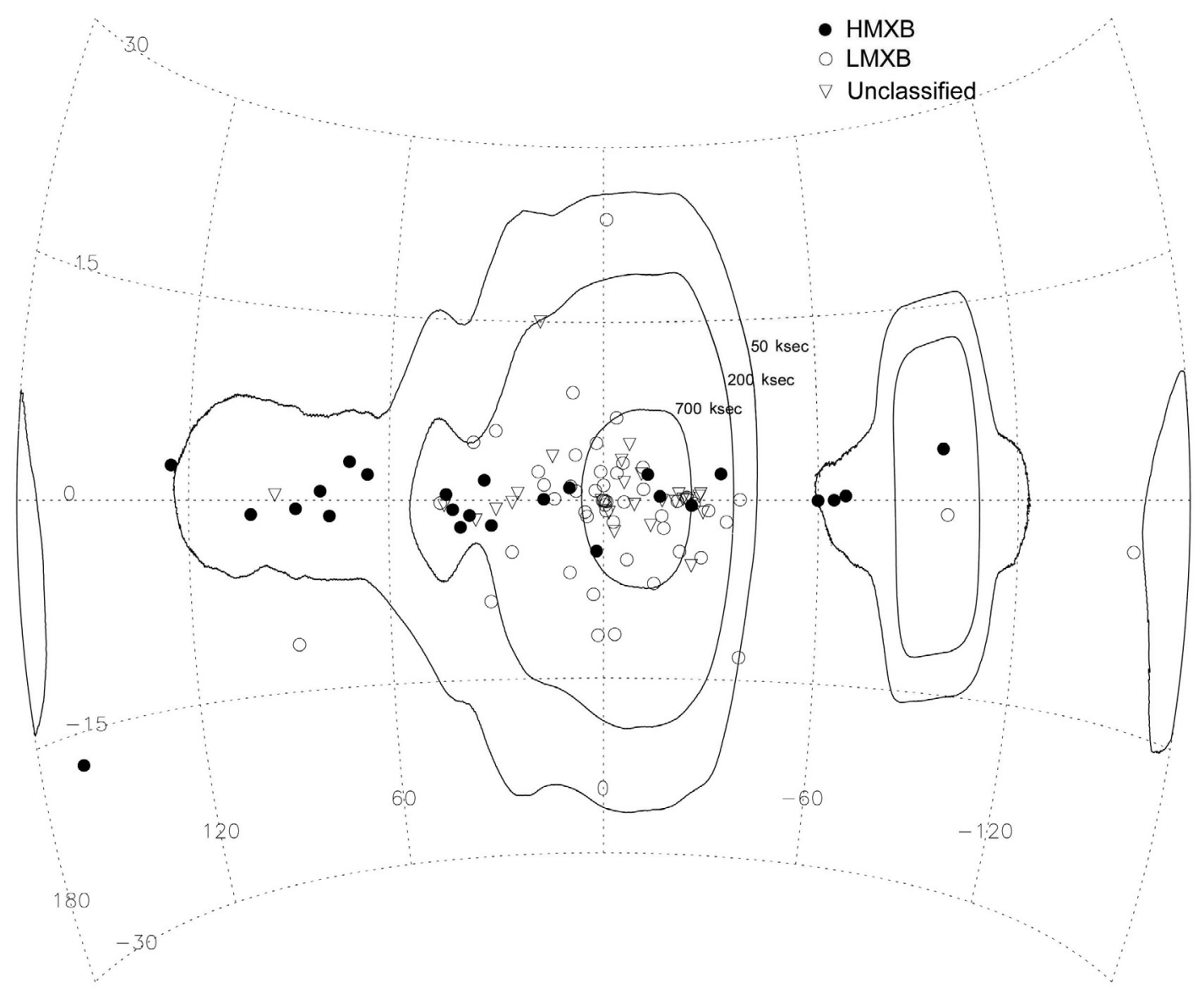

Fig. 5. The galactic distribution of sources in the 1st IBIS/ISGRI catalogue. Filled circles represent HMXBs; open circles represent LMXBs; triangles represent unclassified INTEGRAL sources. Overlaid are contours of exposure time, 50, 200 and $700 \mathrm{ks}$.

photoabsorption generally suffered through the conventional X-ray observations.

\section{The spatial distributions}

The distances to a number of the HMXBs and LMXBs were found through an examination of the available data as published in the literature (White \& van Paradijs 1996; Macomb \& Gehrels 1999; Kuulkers et al. 2003), thus allowing the construction of a 3D model of the source distribution. An updated list of distances collected from available literature for neutron stars and black holes in LMXB as reported by Jonker \& Nelemans (2004) represented one key source of distance information. Different methods were used in deriving the distances of such objects. For neutron stars characterized by the presence of type I bursts, distance can be inferred from observations of Eddington limited bursts. Type I bursts in Low Mass X-ray Binaries are thermonuclear flashes on the neutron star surface (Lewin et al. 1993). During some type I bursts the energy release is high enough such that the luminosity at the surface of the neutron star reaches the Eddington limit (for a typical neutron star the Eddington luminosity value ranges between $2-4 \times$ $10^{38} \mathrm{erg} \mathrm{cm}^{-2} \mathrm{~s}^{-1}$ ). During these events the neutron star's atmosphere expands because of radiation pressure. In this expansion and subsequent contraction the luminosity stay constant near the limit and assuming a pure black body radiation there is a drop in temperature during the expansion of photosphere radius. The enormous number of bursts and new burst sources discovered with the WFCs/BeppoSAX gave a very powerful tool in this respect (see e.g. Cocchi et al. 2001; Kuulkers et al. 2003; in't Zand et al. 2004). In other cases the spectral properties of the companion star have been used. Where possible Jonker \& Nelemans (2004) compared the two different methods and the discrepancies seem to indicate that a larger distance is derived from the first method while using the second approach the distances are underestimated, possibly indicating erroneous spectral classification of the companion star due to fast rotation. For the black hole candidates in our list three different methods have been used to derive distances, namely by using the interstellar absorption properties, from the observed proper motions of receding and approaching lobes when symmetric jets are present, and from the comparison of the absolute magnitude of the donor star with the apparent magnitude using radius, spectral type and luminosity as determined directly from observational data.

A model of the galaxy taken from Vallée (2002) is shown in Fig. 8 with the location of HMXB (filled circles) and LMXB (open circles). It is immediately obvious that the HMXBs and LMXBs do not share the same spatial distribution. The LMXBs 


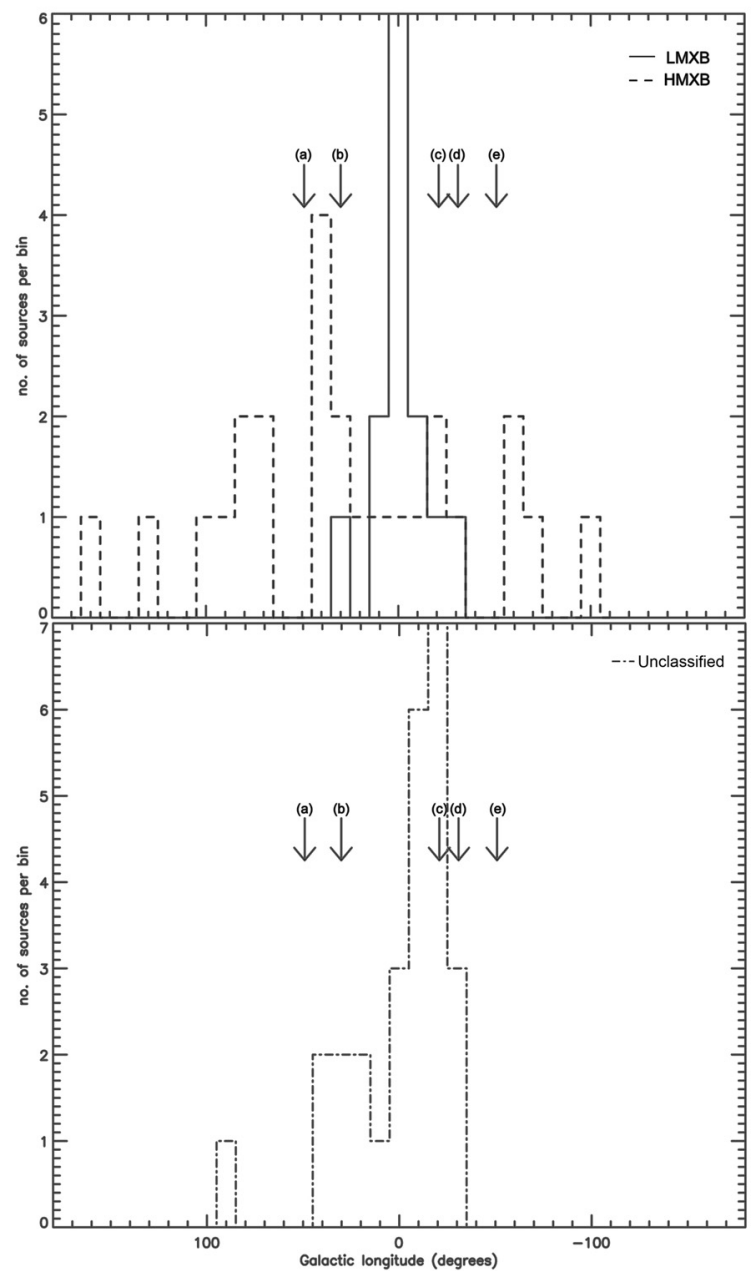

Fig. 6. The Galactic longitude distribution of HMXBs, LMXBs and unclassified sources. Labelled are the locations of the spiral arm tangents: a) - Sagittarius; b) - Scutum; c) - 3-kpc; d) - Norma; e) Centaurus.

can be seen to cluster at the Galactic Centre as implied by their angular distribution discussed in the previous section. The LMXBs are clearly clustered in the central $\sim 3 \mathrm{kpc}$ of the galaxy, while the HMXBs are found outside that radius, in the region associated with the spiral structure. However, for those systems $>3 \mathrm{kpc}$ from the Galactic Centre, it is impossible to say whether LMXB or HMXB follow the spiral structure more strongly. Theoretically the appearance of the HMXB epoch should lag behind the leading edge of spiral arms. This is brought about by the density wave inducing star formation and the HMXB phase subsequently developing some time later. A broad time window is to be expected since the period of elapsed time from star formation to the appearance of a HMXB system is dependent upon the initial masses of its components, and would naturally reflect this spread. For example Tauris \& van den Heuvel (2004) calculate an age of 24.6 million years for a system which starts with $14.4 M_{\odot}$ and $8 M_{\odot}$ stars, and van den Heuvel \& Heise (1972) calculate an age of 15 million years for a system which starts with a $16 M_{\odot}$ and $3 M_{\odot}$ star.

An investigation of this systematic time lag is beyond the scope of this paper. An analysis of this would require a larger

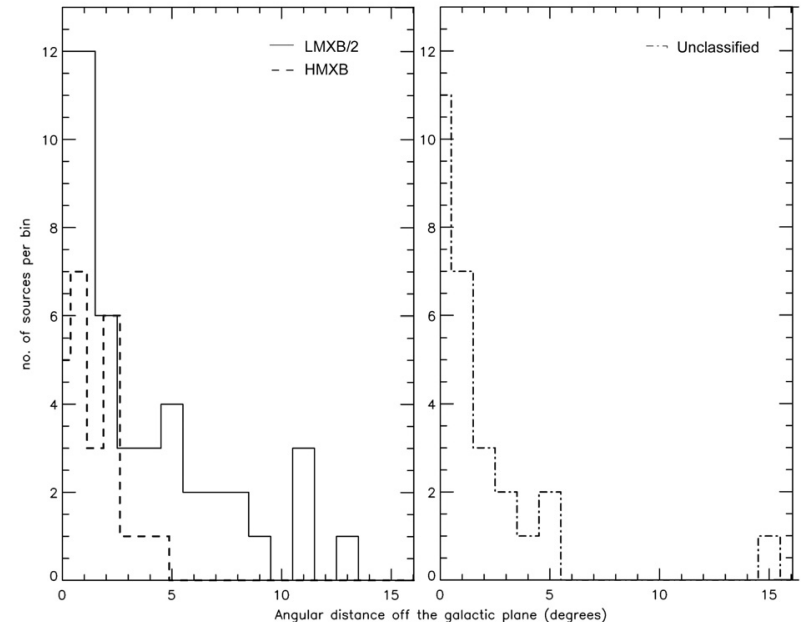

Fig. 7. Angular distribution of sources away from the Galactic Plane. Note that the number of LMXBs is divided by 2 .

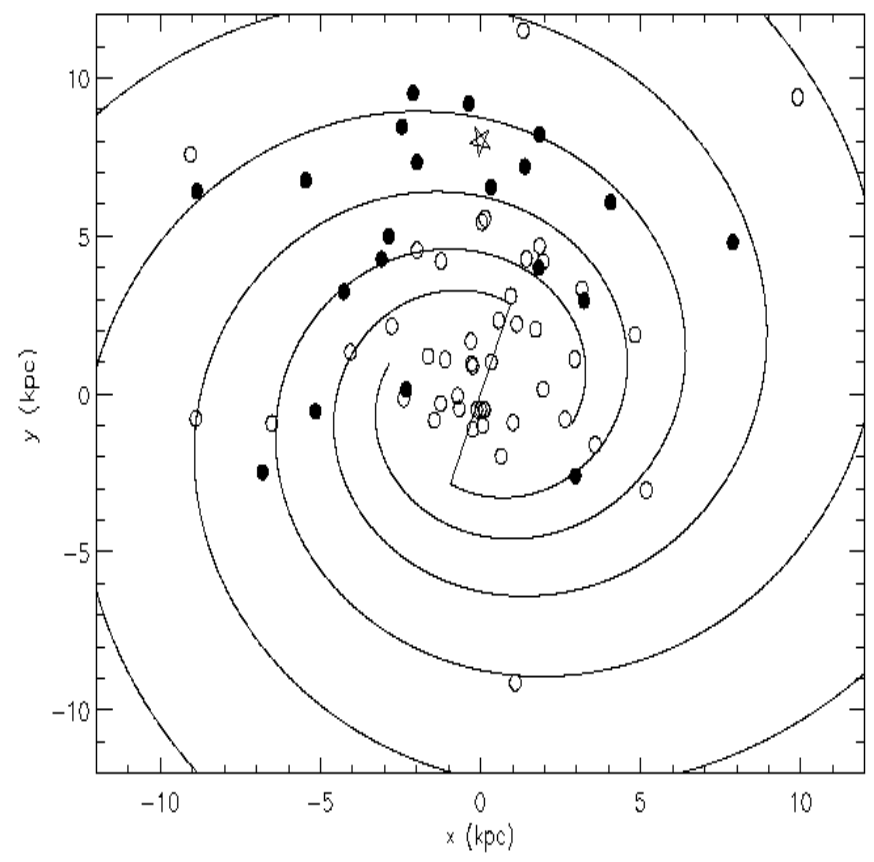

Fig. 8. The galactic distribution of the subset of HMXBs (filled circles) and LMXBs (open circles) for which distance estimates are available in the literature. The Sun is represented by the star symbol and is $8 \mathrm{kpc}$ away from the galactic centre. Superimposed is the 4-arm sprial model of Vallée (2002). Source distance errors are on average of the order of a kpc.

population of HMXBs with well defined distance measures and a model of the galaxy which incorporates the quasi-stationary spiral structure hypothesis proposed by Lin et al. (1969). The key parameter in modelling the spiral structure is $\Omega_{\mathrm{P}}$ the angular velocity of the spiral pattern. Recent measurements of $\Omega_{\mathrm{P}}$ based upon observations of open clusters by Dias \& Lepine (2005) strongly indicate that the spiral pattern rotates as a rigid body with $\Omega_{\mathrm{P}} \sim 25 \mathrm{~km} \mathrm{~s}^{-1} \mathrm{kpc}^{-1}$ at the distance of the Sun from the galactic centre. 

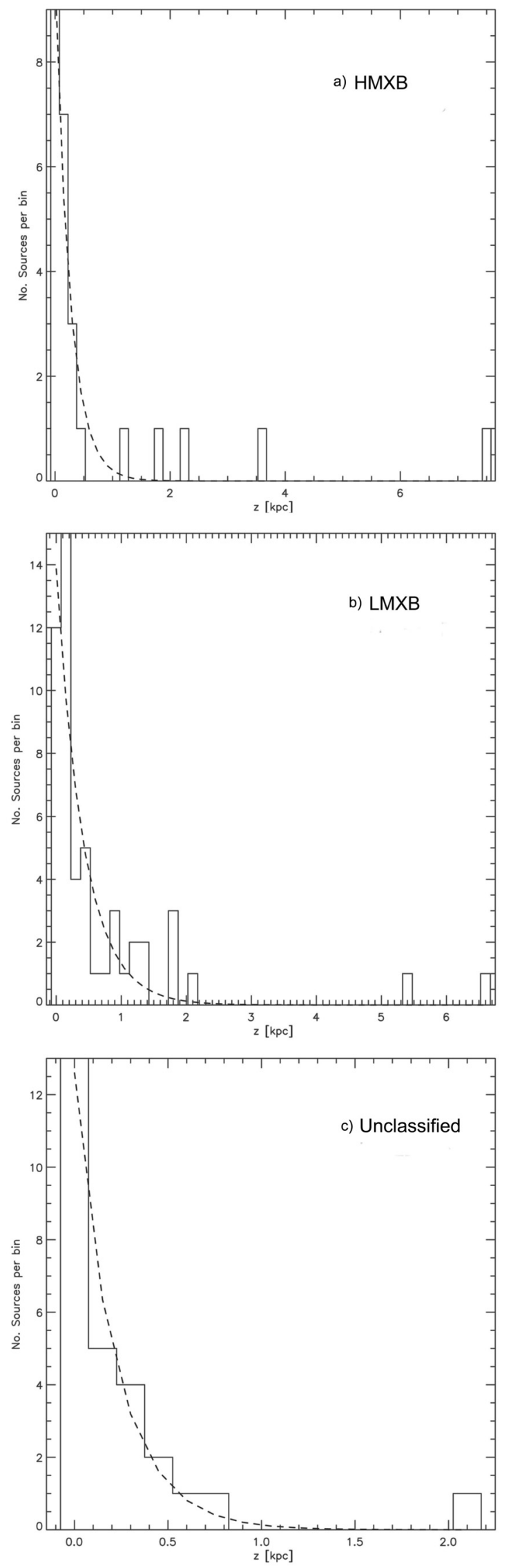

Fig. 9. The distribution of the height off the plane of: a) HMXBs; b) LMXBs; c) unclassified sources. The dashed lines represent exponential fits.
Table 2. Summary of the chracteristics of the principal populations of the IBIS-ISGRI sources in terms of their distributions above the Galactic Plane.

\begin{tabular}{lccc}
\hline \hline & $k$ & $\alpha$ & Scale height (pc) \\
\hline HMXBs & $9 \pm 3$ & $4 \pm 1$ & $240_{-40}^{+90}$ \\
LMXBs & $13 \pm 3$ & $2.9 \pm 0.6$ & $350_{-60}^{+90}$ \\
Unclassified sources & $12 \pm 3$ & $4 \pm 1$ & $240_{-40}^{+90}$ \\
\hline
\end{tabular}

The scale heights of the HMXB and LMXB systems are shown in Figs. 9a and 9b. In each case a simple exponential decrease in numbers as a function of the absolute distance $h$ "above" the galactic plane, $N=k \mathrm{e}^{-\alpha h}$, was used to model the data sets. Making the simplistic assumption that all the unclassified sources reside close to the galactic bulge region, we constructed a pseudo scale height distribution for these unclassified objects as shown in Fig. 9c. Table 2 shows the associated values for the scale height distributions of the three catagories.

It is anticipated that the scale heights of the HMXB and LMXB populations are different, and that the vertical distributions reflect the age disparity of their parent stars. Unfortunately, the precision of the observed scale heights shown in Table 2 is such that the HMXB, LMXB and unclassified sources have scale heights which are within one sigma of each other and are hence indistinguishable. However it is interesting to note that the scale height of this $\gamma$-ray selected HMXB set is consistent with the value of $150 \mathrm{pc}$ found for the HMXB selected from RXTE/ASM data (Grimm et al. 2002). The lack of errors on the HMXB scale height as measured by Grimm et al. (2002) make it difficult to accurately compare the values. Likewise the vertical distribution of the $\gamma$-ray selected LMXBs is compatible with the $410_{-80}^{+100} \mathrm{pc}$ scale height of the RXTE/ASM sample of the LMXB disk population however, our HMXB scale height lies only $\sim 2 \sigma$ from this measurement as well. If we take the unclassified source "scale height" at face value, then it is very interesting to note that, whilst they appear to conglomerate in the galactic bulge region, their vertical distribution is the same as the HMXB systems, reflecting the similarities found in their longitude distributions.

\section{Luminosity functions}

Many of the sources in the first IBIS/ISGRI catalogue have good distance measurements and it is possible to construct their associated luminosity functions. However corrections are required to account for non-uniform exposure, the flux limited nature of the sample and any incompleteness in identifying object classes. This correction is performed by constructing a model of the stellar mass distribution of the galaxy as performed by (Grimm et al. 2002). A galaxy model consisting of a disk and bulge component was constructed using the bulge and disk models of Dehnen et al. (1998):

$\rho_{\text {Bulge }}=\rho_{0, \text { Bulge }}\left(\frac{\sqrt{r^{2}+\frac{z^{2}}{q^{2}}}}{r_{0}}\right)^{-\gamma} \exp \left(-\frac{r^{2}+\frac{z^{2}}{q^{2}}}{r_{\mathrm{t}}^{2}}\right)$ 
$\rho_{\text {Disk }}=\rho_{0, \text { Disk }} \exp \left(-\frac{r_{m}}{r}-\frac{r}{r_{\mathrm{d}}}-\frac{|z|}{r_{z}}\right)$.

Where $\rho_{0 \text {,Bulge }}$ and $\rho_{0 \text {,Disk }}$ are the normalisations, $r$ is the distance in the plane to the galactic centre, $z$ is the distance out of the galactic plane and $R$ is the distance from the galactic centre in spherical coordinates. The other parameters are: $q$ is the oblateness of the bulge; $r_{0}$ is the scale length of the bulge; $r_{\mathrm{t}}$ is the truncation radius of the bulge; $r_{\mathrm{d}}$ is the scale length of the disk; $r_{z}$ is the vertical scale of the disk; $r_{m}$ is the inner disk cut-off. These parameters were taken from fit of RXTE/ASM XRBs to the galactic model by Grimm et al. (2002). However we adjusted $r_{z}$ to match our measured scale heights. All distances are in kiloparsecs. The standard galaxy model uses a mass ratio of 2:1 for disk:bulge. To correct the LMXB population both model components were used whereas the HMXB population uses only the disk component.

To correct the observed luminosity function we calculate the fraction of the galactic mass which is observable at a given luminosity. The correction is taken from Grimm et al. (2002) and is shown below:

$\frac{\mathrm{d} N}{\mathrm{~d} L}=\left(\frac{\mathrm{d} N}{\mathrm{~d} L}\right)_{\mathrm{obs}} \times \frac{M_{\mathrm{tot}}}{M(<D(L))}$.

Where $\frac{\mathrm{d} N}{\mathrm{~d} L}$ is the true luminosity function, $\left(\frac{\mathrm{d} N}{\mathrm{~d} L}\right)_{\text {obs }}$ is the observed luminosity function, $M_{\text {tot }}$ is the total mass of the galaxy, $M(<D)$ is the mass of the galaxy inside a distance $D$ from the Sun. $D(L)$ is defined as:

$D(L)=\min \left(\sqrt{\frac{L}{4 \pi F_{\text {lim }}}}, D_{\max }\right)$.

Where $F_{\text {lim }}$ is the limiting flux of the sample and $D_{\max }$ is the maximum distance from the Sun of the sources used in constructing the luminosity function. We take $F_{\text {lim }}$ as $\sim 5 \mathrm{mCrab}$ in the 20-100 keV energy band which corresponds to $\sim 8.5 \times$ $10^{-11} \mathrm{erg} \mathrm{cm}^{-2} \mathrm{~s}^{-1}$. The maximum distance at which we detect sources approximates to the galactic centre distance. As a result this means that it is only at relatively low luminosities that $D_{\text {max }}$ does not correspond to $D(L)$. As previously discussed the majority of our exposure is in the vicinity of the galactic centre. To compensate for the uneven exposure we modified $F_{\text {lim }}$ for the four quadrants of the galactic plane. As the sensitivity is proportional to the square root of the exposure and that the maximum depth of our sample is $\sim 8 \mathrm{kpc}$ the change in the luminosity function correction within our range of exposures is $<10 \%$

Accreting X-ray binaries are the brightest class of X-ray sources and their emission properties are determined by the nature of the compact object i.e. BHC or neutron star. The strength and geometry of its magnetic field on the neutron stars are also important as is the geometry of the accretion flow from the companion. A neutron star with a strong magnetic field $\left(\sim 10^{12}\right.$ Gauss) will in fact disrupt the accretion flow at several hundred neutron star radii and material is channelled to the magnetic pole. In the case of low magnetic field $\left(<10^{10}\right.$ Gauss $)$ the disk may approach close to the neutron star surface. HMXBs typically have the accreting compact star as a high magnetic field NS or BH and LMXBs have typically

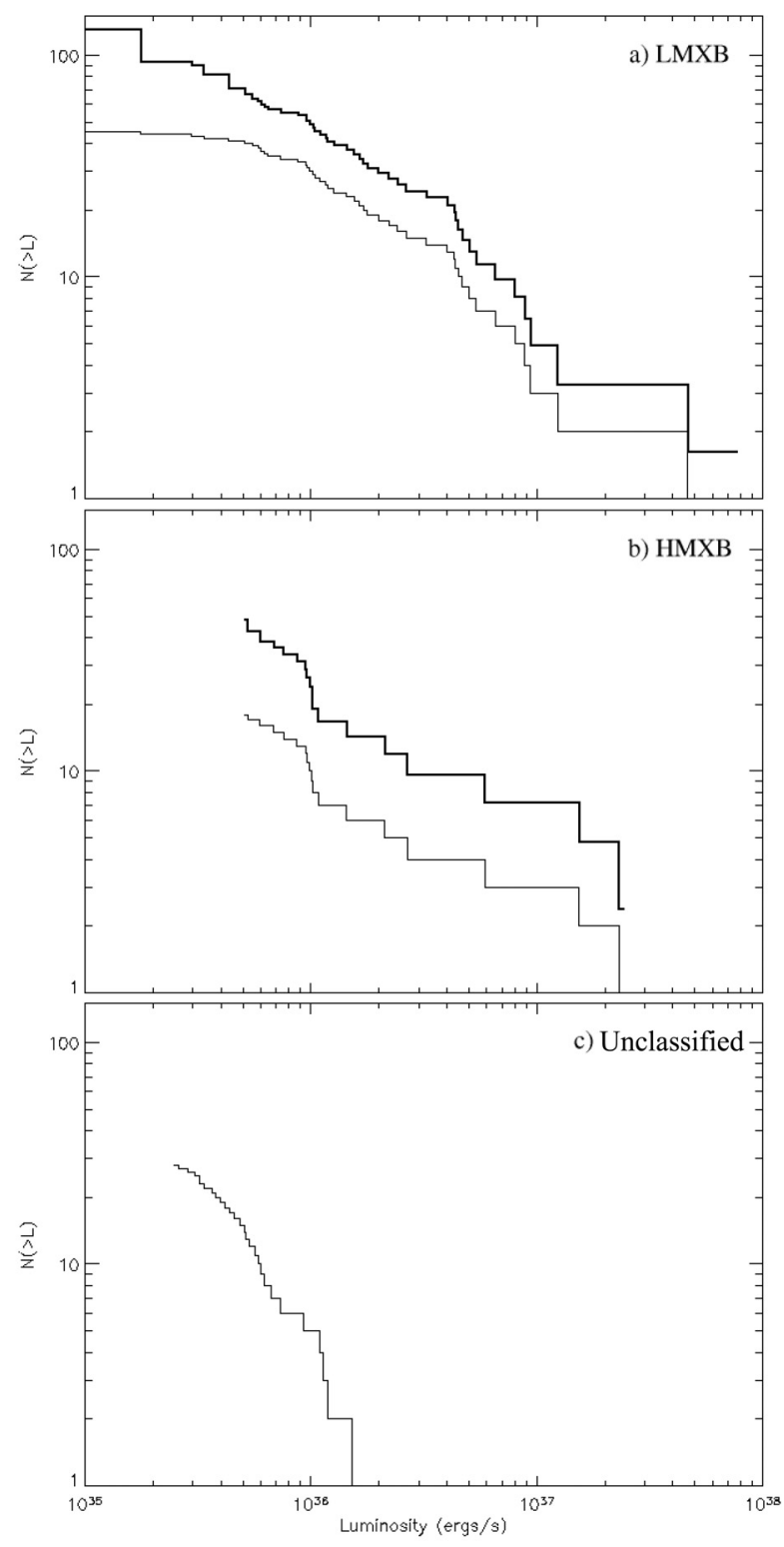

Fig. 10. The 20-100 keV luminosity functions of: a) LMXBs; b) HMXBs; c) unclassified sources. The thin line represents the uncorrected luminosty function. The thick line represents the corrected luminosity function as described in the text. The unclassified sources are uncorrected as their source population is undetermined.

a low magnetic field NS or BH. Strongly or weakly magnetized neutron stars are identified by the presence or absence of pulsations in the light curve of the system. X-ray pulsars are seldom in LMXB, which generally show X-ray bursts that are suppressed when $B$ is $>10^{11}$ Gauss (Lewin \& Joss 1983) and this also explains why bursts are not detected in HMXB systems. For a recent review on accreting neutron star and black hole properties see Psaltis (2004), which also lists the energies of cyclotron lines in accretion powered pulsars used to determine the values for the magnetic field. Magnetic field values for X-ray pulsars in the RXTE/BeppoSAX era are reported in Coburn et al. (2002).

Figure 10 shows the cumulative $\gamma$-ray (20-100 keV) luminosity functions for LMXB, HMXB corrected as outlined in 


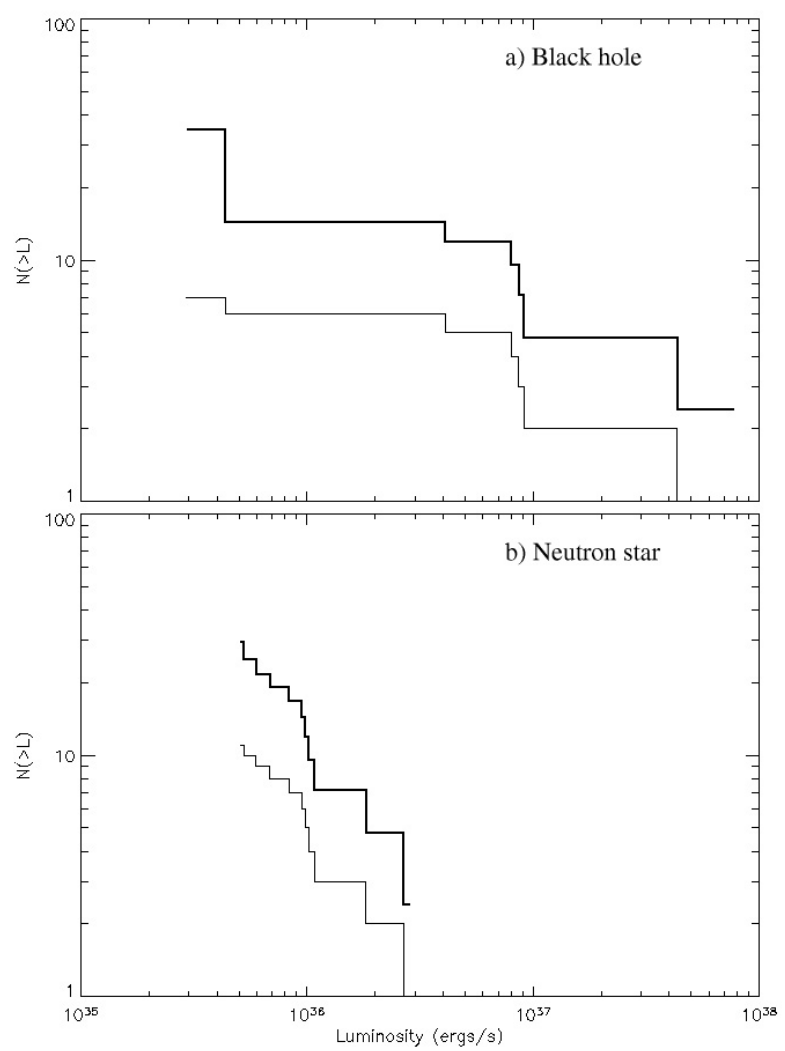

Fig. 11. The $20-100 \mathrm{keV}$ luminosity functions of the IBIS/ISGRI sources separated according to the nature of the compact object: a) black holes; b) neutron stars. The thin line represents the uncorrected luminosty function. The thick line represents the corrected luminosity function as described in the text.

the above text. Also included is a "luminosity function" for the unclassified sources, assuming that they are all at a common distance of $8 \mathrm{kpc}$. It is noticeable that, whereas the low mass systems spread their $20-100 \mathrm{keV}$ luminosities relatively consistently up to and beyond $10^{37} \mathrm{erg} \mathrm{cm}^{-2} \mathrm{~s}^{-1}$, the majority of the high mass systems have a strong inclination to reside around $10^{36} \mathrm{erg} \mathrm{cm}^{-2} \mathrm{~s}^{-1}$. The distribution of the unclassified sources exhibits the low luminosity characteristics of the low mass systems, but also a tendency to favour luminosities close to $10^{36} \mathrm{erg} \mathrm{cm}^{-2} \mathrm{~s}^{-1}$. Whereas Fig. 10 presents the $\gamma$-ray luminosity functions of the known objects selected by the donor system, Fig. 11 shows the luminosity functions of known sources grouped by the nature of the compact object, black hole systems and neutron stars. There are marked differences in their individual distributions. Whereas most of the 20-100 keV luminosities of black hole systems exist around $10^{37} \mathrm{erg} \mathrm{cm}^{-2} \mathrm{~s}^{-1}$, neutron stars do not emit $\gamma$-ray fluxes much in excess of $10^{36} \mathrm{ergs} \mathrm{cm}^{-2} \mathrm{~s}^{-1}$. Although subdividing the classifications has resulted in dwindling numbers of examples and hence statistical significance, it is interesting to note from Figs. 10c and 11, that the unclassified sources are most likely neutron star systems with a significant fraction, but probably not all, cohabiting with high mass companions. The use of a $\sim 10^{37} \mathrm{erg} \mathrm{cm}^{-2} \mathrm{~s}^{-1}$ soft $\gamma$-ray boundary to separate neutron star and black hole systems is not a new concept. Barret et al. (1996) found for high luminosity-high energy emitters, the $\sim 100 \mathrm{keV}$ luminosity of black hole systems is commonly in the range $1-6 \times 10^{37} \mathrm{erg} \mathrm{cm}^{-2} \mathrm{~s}^{-1}$, whereas for neutron star systems it is $\leq 10^{37} \mathrm{erg} \mathrm{cm}^{-2} \mathrm{~s}^{-1}$, as confirmed by INTEGRAL.

\section{Summary and discussion}

The INTEGRAL galactic plane survey and ensuing first catalogue has offered for the first time the possibility to investigate the global $\gamma$-ray characteristics of galactic sources on a reasonable statistical basis. In this paper we have studied the generalised observational features of $\gamma$-ray selected previously unclassified objects, and compared them with the corresponding parameters of known HMXB and LMXB systems. Although the sample of sources has been $\gamma$-ray selected the $\log (N)-\log (S)$, angular and spatial distributions of the known classes, as expected, emulate those of X-ray selected sets e.g. Grimm et al. (2002), there appear to be subtle differences. Of great interest however are the 27 "unidentified" objects, which naturally have overhanging questions such as what are they and how do they fit into mainstream stellar evolution?

Clearly a vigorous programme of follow-up studies on individual objects, using such missions as XMM-Newton, Chandra, and RXTE for timing studies, is one means to pursue the answers to these questions, and such activities are already underway. Walter et al. (2003); Hill et al. (2005); Stephen et al. (2005); Revnivtsev (2003); Matt \& Guainazzi (2003); Rodriguez et al. (2003), and a number of identifications with objects that emit at other wavelengths have been made. The complementary statistical approach employed here essentially comes to the same conclusion i.e. that a large fraction of the unclassified objects are obscured high mass X-ray binary systems. Detailed studies of the main distributional characteristics of the unclassified sources when compared to the equivalent distributions of known X-ray binaries all point in this direction. Their $\log (N)-\log (S)$ and broad angular distributions are consistent with them inhabiting the central 3-4 kpc region of the galaxy, similar to many of the LMXBs. However a closer look at the angular profile shows that it is not the central bulge that determines their finer scale longitude distribution but instead the structure of the inner spiral arms, indicating a considerable number of the unclassified objects are associated with younger stellar systems, and hence probably HMXB. Likewise the galactic latitude distribution of the unclassified sources strongly reflects that of HMXB. More tenuously, the luminosity function of the unclassified sources, assuming them to be close to the galactic centre, exhibits features that are similar to HMXB systems and possibly those incorporating highly magnetised pulsars rather than black holes as the compact companion.

It is hardly surprising that INTEGRAL should discover a population of previously unnoticed sources. The catalogue is $\gamma$-ray selected, and INTEGRAL operates above the energy threshold for which significant photoabsorption takes place in universal abundance matter, so that a strong emitter above $\sim 30 \mathrm{keV}$ can be rendered insignificantly weak in the classical X-ray band. As discussed by Lutovinov et al. (2005a), the HMXB systems constitute the most likely candidates. The stellar wind accretion mechanism that dominates in HMXB systems, as opposed to the Roche lobe overflow associated with 
LMXBs, naturally provides a suitable dense and strongly absorbing circumstellar wind to veil the X-ray emission in a similar manner to the case of the Seyfert 2 AGN configuration (Turner et al. 2000; Malizia et al. 2003).

Exactly how the $\gamma$-ray selected highly absorbed systems fit into the overall picture of binary star evolution is currently unclear. This is principally due to the requirement of a suitable companion able to provide a sufficiently strong stellar wind mass loss rate $\left(\dot{M} \geq 10^{-4} M_{\odot} \mathrm{yr}^{-1}\right)$ that is capable of generating a suitably dense gas surrounding the compact object to stifle the X-ray emission. We need to understand why they are different to "normal" HMXB. Do these highly absorbed systems relate to the mass/giant nature of the primary star, or to the orbit configuration, or are they experiencing a phase the binary systems routinely pass through; but have not been exposed at other wavelengths? Do they need to be HMXB? Clearly a series of dedicated observations are required to solve this problem. If they are not all HMXB systems, then could some of them be intermediate mass X-ray binaries (IMXBs) (van den Heuvel 1975). Some of the sources could be systems that are passing through, or close to the common envelope phase (Iben \& Livio 1993; Taam \& Sandquist 2000). However on statistical grounds this seems unlikely due to the requirement that their relative numbers need to compatible with the relatively short timescale $\left(\leq 10^{3} \mathrm{yr}\right)$ of this phase, which is dictated by the drag force created as the compact star moves through the envelope of the extended companion.

Clarification of the above general issues raised by the INTEGRAL source discoveries will also facilitate a better understanding of the relative production of neutron stars and black holes. There is uncertainty in the threshold mass for core collapse into a black hole. Lower mass helium star systems should also be considered (Brown et al. 1999). A fundamental aspect in this context is whether, during the mass loss period, the helium core remains surrounded by a thick hydrogen mantle, so that common envelope evolution proceeds (Nelemans \& van den Heuvel 2001). It is also possible that a neutron star spiralling inwards in a common envelope might rapidly accrete and eventually collapse into a black hole (Chevalier et al. 1993). On a statistical basis INTEGRAL will provide a clearer picture of the relative numbers of the various sub species of $\mathrm{X}$-ray binary systems, and consequently a more balanced understanding of the rather murky final stages of binary star evolution and by implication eventually more reliable estimates for the relative numbers of black hole and neutron stars.

Acknowledgements. This work is based on observations obtained with INTEGRAL an ESA science missions with instruments, science data centre and contributions funded by the ESA member states with the participation of the Czeck Republic, Poland, Russia and the USA.

The Southampton group acknowledge support through PPARC research grants. A.B.H. acknowledges funding support from a PPARC Ph.D. studentship. A.B., L.B., J.B.S. and P.U. acknoweledge the Italian Space agency financial support via contract I/R/046/04.

\section{References}

Barret, D., McClintock, J. E., \& Grindlay, J. E. 1996, ApJ, 473, 963 Bird, A. J., Barlow, E. J., Bassani, L., et al., 2004, ApJ, 607, L33 Brown, G. E., Lee, C. H., \& Bethe, H. A. 1999, New Astron., 4, 313
Chevalier, R. A. 1993, ApJ, 411, L33

Coburn, W., Heindl, W. A., Rothschild, R. E., et al. 2002, ApJ, 580, 394

Cocchi, M., et al. 2001, Proceeding of the 4th INTEGRAL Workshop, ESA-SP-459, 279

Dehnen, W., \& Binney, J. 1998, MNRAS, 294, 429

Dias, W. S., \& Lepine, J. R. D. 2005 [arXiv:astro-ph/0503083]

Englmaier, P., \& Gerhard, O. 1999, MNRAS, 304, 512

Feast, M., \& Whitelock, P. 1997, MNRAS, 291, 683

Grimm, H.-J., Gilfanov, M., \& Sunyaev, R., 2002, A\&A, 391, 923

Gros, A., Goldwurm, A., Cadolle-Bell, M., et al. 2003, A\&A, 411, L179

Hertz, P., \& Grindlay, J. 1984, ApJ, 278, 137

Hill, A. B., Walter, R., Knigge, C., et al. 2005 A\&A, 439, 255

Iben, Jr. I., \& Livio, M. 1993, PASP, 105, 1373

in't Zand, J. M, Verbunt, F., \& Heise, J. 2004, NuPhS, 132, 486

Jonker, P. G., \& Nelemans, G. 2004, MNRAS, 354, 355

Koyama, K., Kamada, M., Kunieda, H., et al. 1990, Nature, 343, 148

Kuulkers, E., den Hartog, P. R., in't Zand, J. J. M., et al. 2003, A\&A, 399, 663

Lewin, W. H. G., \& Joss, P. C. 1983, in Accretion driven stellar X-ray sources, ed. W. H. G. Lewin \& E. P. J. van den Heuvel, Cambridge University, 41

Lewin, W. H. G., van Paradijs, J. \& Taam R. E. 1993, Space Sci. Rev., 62,223

Lin, C. C., \& Shu, F. H. 1964, ApJ, 140, 646

Lin, C. C., Yuan, C., \& Shu, F. H. 1969, ApJ, 155, 721

Liu, Q. Z., van Paradijs, J., \& van den Heuvel, E. P. J. 2000, A\&AS, 147, 25

Liu, Q. Z., van Paradijs, J., \& van den Heuvel, E. P. J. 2001, A\&A, 368,1021

Lutovinov, A., Revnivtsev, M., Molkov, S., et al. 2005a, A\&A, 430, 997

Lutovinov, A., Revnivtsev, M., Gilfanov, M., et al. 2005b, A\&A, accepted [arXiv:astro-ph/0411550]

Macomb, D. J., \& Gehrels, N. 1999, ApJS, 120, 335

Malizia, A., Bassani, L., Stephen, J. B., et al. 2003, ApJ, 589, L17

Matt, G., \& Guainazzi, M. 2003, MNRAS, 341, L13

Murdoch, H. S., \& Crawford, D. F. 1973, ApJ, 183, 1

Nelemans, G., \& van den Heuvel, E. P. J. 2001, A\&A, 376, 950

Psaltis, D. 2004, in Compact Stellar X-ray Sources, ed. W. H. G. Lewin \& M. van der Klis

Revnivtsev, M. G. 2003, Astro. Lett., 29, 644

Rodriguez, J., Tomsick, J. A., Foschini, L., et al. (2003), A\&A, 407, L41

Stephen, J. B., Bassani, L., Molina, M., et al. 2005, A\&A, 432, L49

Sugizaki, M., Mitsuda, K., Kaneda, H., et al. 2001, ApJS, 134, 77

Taam, R. E., \& Sandquist, E. L. 2000, ARA\&A, 38, 113

Tauris, T. T., \& van den Heuvel, E. J. P. 2004 [arXiv: astro-ph/0303456]

Taylor, J., \& Cordes, J. 1993, ApJ, 411, 674

Turner, T. J., Perola, G. C., Fiore, F., et al. 2000, ApJ, 531, 245

Ubertini, P., Lebrun, F., Di Cocco, G., et al. 2003, A\&A, 411, L131

Vallée, J. P. 2002, ApJ, 566, 261

van den Heuvel, E. J. P., \& Heise, J. 1972, Nature, 239, 67

van den Heuval, E. P. J. 1975, ApJ, 198, L109

van Paradijs, J., \& White, N. 1995, ApJ, 447, L33

Warwick, R. S., Marshall, N., Fraser, G. W., et al. 1981, MNRAS, 197, 865

Walter, R., Rodriguez,J., Foschini, L., et al., 2003, A\&A, 411, L427

White, N. E., \& van Paradijs, J. 1996, ApJ, 473, L25

Winkler, C., Courvoisier, T. J.-L., Di Cocco, G., et al. 2003, A\&A, 411, L1 